\title{
Association between Fitness, Different Indicators of Fatness, and Clustered Cardiovascular Diseases Risk Factors in Portuguese Children and Adolescents
}

\author{
Clarice L. Martins ${ }^{1, *}$, Lars Bo Andersen $^{2,3}$, Luísa M. Aires ${ }^{1}$, José C. Ribeiro ${ }^{1}$ and Jorge A. Mota ${ }^{1}$ \\ ${ }^{1}$ Faculty of Sports, Porto University, Research Centre in Physical Activity, Sports and Leisure, Porto \\ ${ }^{2}$ Institute of Sport Sciences and Clinical Biomechanics, University of Southern Denmark, Denmark \\ ${ }^{3}$ Department of Sports Medicine, Norwegian School of Sport Sciences, Norway
}

\begin{abstract}
Objectives: To investigate the relationship between cardiovascular diseases risk factors, cardiorespiratory fitness and three different indicators of fatness, and investigate if these relationships are independent by each other.

Methods: 491 children and adolescents were evaluated. Standardized metabolic risk scores were computed. Multiple linear regression and Univariate Analysis of Variance - GLM were used.

Results: Fit youngsters presented a better profile for each of the risk factors. Belonging to the unfit category increased the risk of having high MRS. After adjustments for fatness indicators, the relationship between fitness and MRS disappeared, and obesity indicators presented significant relationship with the MRS.
\end{abstract}

Conclusion: Fitness and fatness are associated with clustered risks by different pathways.

Keywords: Fitness, fatness, children and adolescents.

\section{INTRODUCTION}

Clustering of CVD risk factors is known as the coexistence of several risk factors in the same subject [1]. There is a multiplicative effect of the biological risk factors when they occur together for a particular subject [2], which may have higher clinical relevance. Furthermore, CVD risk factors clustering have been identified in children and adolescents [3].

CRF is a direct marker of physiological condition, reflecting the capacity of the cardiovascular and respiratory systems to provide oxygen during a continuous physical activity, carrying out prolonged exercises [4]. Recent studies have shown that not only obesity [5] or physical activity [6] but also cardiorespiratory fitness (CRF) should be studied when analyzing the prevalence of CVD risk factors in youth population. Indeed, while some observational studies have shown that childhood obesity is associated with a higher metabolic risk profile [7], results from other surveys such as the Quebec Family Study indicated that in different BMI categories, higher levels of CRF were associated with a better CVD risk profile [8]. Further, in Danish children it was suggested a strong relationship between activity and metabolic risk in children with low cardiorespiratory fitness [9].

Although an inverse association between obesity and levels of CRF has been suggested [10], there is little evidence

*Address correspondence to this author at the Faculty of Sports, Porto University, Research Centre in Physical Activity, Sports and Leisure, Porto; Tel: +351 919896911; Fax: +351 229825331;

E-mail: clarice.br@fade.up.pt showing an interaction between $\mathrm{CRF}$ and fatness in relation to CVD risk factors [6]. Moreover, there is some evidence that youngsters with a high CRF profile have a healthier cardiovascular profile not only during adolescence but also in later life [11], and this evidence seems to be independent of body weight [12]. Therefore, based on this scarce data about the inter-relationship between CRF and adiposity, some studies are attempting to determine the contribution of each variable on CVD risk factors by controlling for the simultaneous effect of the other variable [13].

Abdominal fat and low CRF may both increase the risk of clustered CVD risk. It may therefore be of value to describe the independent association of these traits in relation to clustering of CVD risk factors.

Therefore, the aim of the present study was 1 . to investigate the relationship between cardiovascular risk factors, CRF and three different indicators of fatness (BMI; \%Fat and wais circumference), and 2. investigate if these relationships are independent by each other.

\section{SUBJECTS AND METHODS}

\section{Design and Sample}

This study was carried-out as a part of an observational research project looking at the prevalence of CVD risk factors and levels of physical fitness in children and adolescents aged 10-16 years-old of both genders. The study was conducted at Porto district, Portugal. Children and adolescents were chosen at random from the $4^{\text {th }}$ till the $12^{\text {th }}$ school grade, according to general school system rules, which has previously been described [14]. 
From a total of 516 students that agreed to participate in the study, a total of 491 children did all measurements (223 boys, 45,4\% and 268 girls, 54,6\%). Children, parents and schools approved the study protocol.The nature, benefits, and risks of the study were explained to the volunteers, and parents written informed consent was obtained before the study, consistent with the Helsinki Declaration. The evaluation methods and procedures were approved by the Scientific Board of the Faculty of Sports of the University of Porto.

All measures were carried out by a specialized group (Physical Education teachers, medical doctor).

\section{Daily Evaluation Protocol}

Subjects were identified through his/her code number and code of the school. Fasting blood samples were taken followed by blood pressure measurements. The children were then given breakfast followed by the determination of their maturational stage. Finally the shuttle-run test was performed. The variables were measured between 8:00 and 11:00am.

\section{Blood Sampling}

Capillary blood samples of participants were taken from the right earlobe after at least 12 hours fasting in order to obtain values of plasmatic total cholesterol (TC), high density lipoprotein cholesterol (HDL) and fasting glucose (GLUC). The blood samples were drawn in capillary tubes (33 $\mu$ l, Selzer) coated with lithium heparin and immediately assayed using Colestech LDX® Analyser.

The sample was applied into a Cholestech LDX® cassette and the analyser separates the plasma and the blood cells. Cassettes were stored in the refrigerator after reception. The Cholestech LDX $®$ analyser has been proven to provide good agreement with laboratory measures for populationbased screaming for cardiovascular risks factors [15].

\section{Blood Pressure}

Blood pressure (BP) was measured using the Dinamap adult/pediatric and neonatal vital signs monitors, model BP8800. Measurements were taken by a trained technician and with all children sitting after at least $5 \mathrm{~min}$ rest. Two measurements were taken after five and ten minutes rest. The mean of these two measurements was used for statistical analysis. If the two measurements differed by $2 \mathrm{mmHg}$ or more the protocol was repeated (two new measurements until the difference did not exceed $2 \mathrm{mmHg}$ ).

\section{Anthropometric Measures and Body Fat}

Body height was measured to the nearest $\mathrm{mm}$ in bare or stocking feet with the adolescent standing upright against a Holtain Stadiometer. Weight was measured to the nearest 0.1 $\mathrm{kg}$, lightly dressed and after having breakfast, using an electronic weight scale (Seca 708 portable digital beam scale). Body mass index (BMI) was calculated from the ratio of body weight $(\mathrm{kg}) /$ body height $\left(\mathrm{m}^{2}\right)$.

To evaluate the waist circumference (WC), the NHANES [16] protocol was used. A bony landmark is first located and marked. The subject stands and the examiner, positioned at the right of the subject, palpates the upper hip bone to locate the right iliac crest. Just above the uppermost lateral border of the right iliac crest, a horizontal mark is drawn, and then crossed with a vertical mark on the midaxillary line. The measuring tape is placed in a horizontal plane around the abdomen at the level of this marked point on the right side of the trunk. The plane of the tape is parallel to the floor and the tape is snug, but does not compress the skin. The measurement is made at a normal minimal respiration

Body fat was determined by tricipital and subscapular skinfolds, according to Heyward [17]. Each skinfold was measured twice and in a successive way, in the right side of the body. However if in these two measurements there was a difference above $5 \%$ a third measure was performed. The final result consisted of the mean of the two or three measurements for each skinfold. A Harpender caliper with a constant pressure of $10 \mathrm{~g} / \mathrm{mm}^{2}$ was used and all measurements were completed by the same observer. The percentage of fat (\%fat) was estimated from skinfolds measurements, according to Slaughter et al. [18] equations.

\section{Maturational Stage}

Regarding the maturational stage, the adolescents were inquired separately during physical examination. Each subject self-assessed his/her stages of secondary sex characteristics. Stage of breast development in females and pubic hair in males was evaluated according to the criteria of Tanner [19]. Previous study showed a correlation of 0.73 between ratings on two occasions (three day interval) in a sub-sample of 50 selected subjects and concordance between selfassessments of sexual maturity status and physician assessment ranged from $63 \%$ for girls and $89 \%$ for boys [20]s.

\section{Cardiorespiratory Fitness (CRF)}

CRF was predicted by maximal multistage $20 \mathrm{~m}$ shuttlerun test according to procedures described by Léger et al. [21]. The FITNESSGRAM test battery [22] which comprehends several physical fitness tests was selected because of its easy of administration to large numbers of subjects, and in addition its choice of reliable and valid health-related physical fitness measures [23]. From the tests that compound the FITNESSGRAM, only The Shuttle Run Test, which predicts maximal aerobic capacity according to the number of completed laps, was obtained. Furthermore, the 20 meter Shuttle Run Test showed good correlation with directly measured $\mathrm{VO}_{2} \max (\mathrm{r}=0.80)$ suggesting that it could be used as a measure of aerobic fitness in children [24]. Nevertheless, $\mathrm{VO}_{2}$ max expressed per unit body mass $\left(\mathrm{ml} \cdot \mathrm{kg}^{-1} \cdot \mathrm{min}^{-1}\right)$ has been criticized [25]. Therefore, the CRF was expressed per number of completed laps achieved in the Shuttle Run Test. There are several studies that assessed cardiorespiratory fitness by the number of completed laps in Shuttle-Run Test [26].

Children were then categorized in fit or unfit according to adopted age-adjusted criterion referenced health standards (Health Fitness Zone) for individual CRF test item in the Fitnessgram test battery, as belonging to a healthy zone (fit) or under a healthy zone (unfit).

\section{Statistical Analysis}

Descriptive statistics were used in order to characterize the sample. Given that fact, there is not a clinical criteria for the metabolic syndrome and in the literature different defini- 
Table 1. Descriptive Statistics. Differences Between Fit and Unfit Groups

\begin{tabular}{|c|c|c|c|c|c|c|}
\hline \multirow[b]{2}{*}{ Variables } & \multicolumn{2}{|c|}{ Fit } & \multicolumn{4}{|c|}{ Unfit } \\
\hline & $\mathbf{N}$ & Mean (SD) & $\mathbf{N}$ & Mean (SD) & $\mathbf{t}$ & $\mathbf{p}$ \\
\hline Age & 206 & $13.48(.13)$ & 208 & $14.42(.14)$ & 4.824 & NS \\
\hline Weight & 206 & $54.09(12.0)$ & 208 & $55.00(10.7)$ & .802 & NS \\
\hline Height & 206 & $161.33(11.5)$ & 208 & $159.36(8.1)$ & -2.009 & .045 \\
\hline BMI & 206 & $20.55(2.8)$ & 208 & $21.53(3.3)$ & 3.294 & .001 \\
\hline Completed laps & 189 & $51.51(21.3)$ & 208 & $26.17(9.9)$ & -14.923 & .000 \\
\hline $\mathrm{TC}$ & 206 & $145.13(25.7)$ & 208 & $150.61(25.8)$ & 2.164 & .031 \\
\hline HDL & 206 & $46.00(11.1)$ & 208 & $43.69(11.0)$ & 2.110 & .035 \\
\hline LD1 & 206 & $90.85(23.8)$ & 208 & $92.54(24.2)$ & .714 & NS \\
\hline Trigl & 206 & $52.94(15.0)$ & 208 & $60.40(21.1)$ & 4.143 & .000 \\
\hline Glucose & 206 & $84.17(7.3)$ & 208 & $85.41(7.1)$ & -1.753 & NS \\
\hline $\mathrm{BP}$ & 206 & $141.25(17.2)$ & 208 & $142.74(15.7)$ & .922 & NS \\
\hline WC & 206 & $73.29(8.0)$ & 208 & $75.84(8.8)$ & 3.073 & .002 \\
\hline$\%$ fat & 205 & $19.90(6.3)$ & 207 & $26.52(8.2)$ & 9.228 & .000 \\
\hline MRS & 206 & $-.035(.46)$ & 208 & $.029(.48)$ & 1.390 & NS \\
\hline
\end{tabular}

Metabolic Risk Score (TC, HDL, LDL, Triglycerides, Glucose, BP).

tions differ in detail and inclusion criteria [27], none of its cutoff points apply specifically to children [28].

There are two reasons for not using cut off points when we constructed the composite score: 1) no consensus about the level of the cut off points, and 2) it reduces information to use cut offs instead of continuous scores.

Standardized metabolic risk scores (MRS) were computed for each risk factor. The following variables were included in the MRS: glucose, HDL-C, LDL-C, TC, blood pressure (systolic and diastolic), and triglycerides. Each of these variables was standardized as follows: standardized value $=($ value - mean $) /$ standard deviation. The HDL-C standardized values were multiplied by -1 to confer higher risk with increasing value for the purpose of calculating the MRS, which was obtained as the mean of the 6 standardized scores. This approach has been used before in youth population [29]. Multiple linear regression analysis was used in order to investigate the relationships between fatness, fitness and the MRS. Four independent variables (WC, \%FAT, BMI, and CRF) and two dependent variables (MRS and $\mathrm{CRF}$ ) were performed in separate models.

The Univariate Analysis of Variance - GLM was used to determine if different levels of fitness (Unfit and Fit) were related with clustered metabolic disorders, independent of fatness indicators (Zscore of BMI, WC and \% fat). Four different models were analyzed. In the first model, the influence of CRF in the MRS, without obesity indicators was analyzed. The three subsequent models indicate the influence of both CRF and obesity indicators in the MRS. For each model, two analyses were done. In the first one, a crude metabolic risk score was constructed and the analysis was adjusted for sex and maturational status. As there was no interaction between the terms, age and sex specific standardized metabolic risk scores (MRS) were computed and all subjects analyzed together. Analysis was performed with the statistical software package SPSS 15.0 for Windows and level of significance was set up at $\mathrm{p} \leq 0.05$.

\section{RESULTS}

Table 1 shows descriptive statistics (mean \pm SD) of all variables, separately for fit and unfit subjects. It was observed that in general, unfit subjects tend to have higher mean values of metabolic risk factors, especially the lipid profile. Total cholesterol, HDL cholesterol and triglycerides levels different between groups. Unfit children presented higher values for glucose and LDL cholesterol as well, though this tendency was not significant.

Regarding the adiposity indicators, fit subjects presented significant lower values for BMI, waist circumference and percentage of fat, when compared to unfit.

Relationships between the MRS, the three indicators of fatness and fitness, and the relationship between fitness and fatness indicators are shown in Table 2. All the variables were expressed as z-score values. In this analysis, significant relationships between the MRS, fat indicators and fitness were observed. The strongest association was observed when MRS and fitness were related to percentage of fat $(\beta=.089$ and $\beta=-.317$ respectively).

In Table 3 four different models of predicting metabolic disorders were analyzed by an univariate linear regression model. The main outcome was the MRS and the independent variables were fitness, BMI, WC and \% fat. The first model 
Table 2. Relationships Between the MRS, Fitness and Fatness

\begin{tabular}{|l|c|c|c|c|c|c|}
\hline \multirow{2}{*}{ Variables } & \multicolumn{3}{|c|}{ MRS } & \multicolumn{2}{c|}{ Fitness } \\
\cline { 2 - 7 } & $\beta$ & $\mathrm{Sig}$ & $\mathrm{CI}(95 \%)$ & $\beta$ & Sig & \multicolumn{1}{c|}{ CI $(95 \%)$} \\
\hline WC & .084 & .003 & $(.028 ; .139)$ & -.123 & -010 & $(-.217 ;-.029)$ \\
\hline$\% \mathrm{FAT}$ & .089 & .000 & $(.040 ; .139)$ & -.317 & -.000 & $(-.396 ;-.239)$ \\
\hline BMI & .081 & .001 & $(.034 ; .128)$ & - & -001 & $(-.217 ;-.053)$ \\
\hline Fitness & -.353 & .031 & $(-.675 ;-.032)$ & & - \\
\hline
\end{tabular}

Multiple linear regressions adjusted for sex and maturational status

$* \mathrm{p} \leq 0.05$

Table 3. Fitness as Predictor of Clustered Risk Factors. All Models Adjusted for Sex and Maturational Status, With Further Adjustments for Obesity Indicators

\begin{tabular}{|l|c|c|c|c|}
\hline \multicolumn{2}{|c|}{ Models } & F & p I (95\%) & $(, 020 ;, 296)$ \\
\hline \hline Model 1 & Unfit & .158 & .025 & $(-, 004 ;, 268)$ \\
\hline \multirow{2}{*}{ Mode1 2 } & Unfit & .132 & .058 & $(, 017 ;, 040)$ \\
\cline { 2 - 5 } & BMI & .033 & .000 & $(-, 014 ;, 261)$ \\
\cline { 2 - 5 } Model 3 & Unfit & .123 & .079 & $(, 004 ;, 016)$ \\
\hline \multirow{2}{*}{ Model 4 } & WC & .010 & .001 & $(-, 047 ;, 235)$ \\
\cline { 2 - 5 } & Unfit & .094 & .000 & $(, 007 ;, 020)$ \\
\hline
\end{tabular}

Dependent Variable: Metabolic risk score; Fit health zone is the reference category

Model 1 = fitness; Model 2 = fitness and BMI; Model $3=$ fitness and WC; Model $4=$ fitness and \%fat

$* \mathrm{p} \leq 0.05$

shows fitness as predictor of MRS and indicates that the differences between the fit and the unfit group was $F=.158$. The other three models represent the same idea, with further adjustment for BMI, WC and \%fat, respectively.

The results indicate that after adjustment for fat, the relationship between fitness and MRS disappears for all the three adiposity indicators.

\section{DISCUSSION}

This study analyzes whether the association between fitness and CVD is independent of fatness.

Since the onset of chronic disease risk factors lies in early childhood, it is of great importance to examine the potential risk in order to make effective preventive strategies targeting those at risk as early as possible. It is important to point out that interventions targeting fitness may change fitness rapidly while interventions targeting fatness may take longer time before major effects are seen.

The main outcome of this study was that both fitness and fatness were associated with clustering risk factors in children and adolescents.

Although our data showed that our fit youngsters presented a better data with regard each of CVD risk factors analyzed isolated (Table $\mathbf{1}$ ). The results of the regression analysis
(Table 3) showed that belonging to the unfit category increased the risk of having high MRS $(B=.158 ; \mathrm{p}<0.05)$. However, when models were adjusted for each of the indicators of adiposity (BMI, \%FAT; WC), the relationship between being fit and MRS disappeared. All the three fat indicators were associated with MRS, after adjustments for gender, age and maturation. Thus, our findings agree with similar studies showing that higher levels of CRF are inversely associated with healthier CVD profile in children and adolescents when CVD risk factors were computed as a clustered metabolic risk score $[6,30]$.

We observed a statistically significant association between CRF and MRS. Though, after adjustment for adiposity, this association disappeared. This observation could let to the interpretation of fitness not being important to this relationship between fitness, fatness and MRS. However, this may not be true. The interpretation depends on how the causal chain is. If fatness is an intermediate link between low fitness and CVD risk then the fitness would disappear after adjustment for fatness, but low fitness would still be the cause.

Overweight and obesity are associated with an increased risk of CVD risk factors early in life. Our data seems to confirm that adiposity, regardless the indicator used, is a strong predictor of MRS. Reinforcing this idea, Eisenmann et al. [5] 
resented some evidences for considering not only obesity, but both adiposity indicator and CRF when interpreting CVD risk factors in the young population. Some data pointed out that moderate to higher levels of CRF have been associated with lower abdominal adiposity, suggesting that a mechanism might exist by which CRF attenuates the health risk of obesity [31]. The association between fitness and MRS changed when adjusted for fatness could support the hypothesis that MRS is not entirely mediated by fitness or fatness, but only part of this association is mediated by a singular factor.

In addition, obesity has been shown to be strongly associated with insulin resistance [32] and other CVD risk factors. When evaluating the relation between CRF and insulin sensitivity in U.S youth, Imperatore et al. [33] observed that in boys, higher CRF was associated with high insulin sensitivity, independent of BMI. In girls this association disappeared after controlling for BMI. Given that insulin resistance could be a predictor of obesity and cardiovascular risk factors [34], the findings of these studies could explain some of our results and suggest that maybe the insulin resistance could be the mediator link between CRF, obesity and clustered risk factors. If we assume that fatness is the main mediator of the relationship between clustered risk factors and CRF [35] we do not take in consideration the fact that insulin resistance could be the link behind this relation. Further, insulin sensitivity is mainly related to the muscle tissue, because a great portion of the carbohydrate is stored or burned in the muscle [36]. One-leg training models have shown that insulin sensitivity is very local. Glucose uptake can be doubled in the trained leg compared to the untrained leg, and this is independent of fatness (the legs share the rest of the body) [37].

All those studies corroborate in some sense with our findings and highlight the important need of increasing the levels of CRF in children and adolescents in order to minimize the prevalence of CVD risk factors in this population. Nevertheless, some limitations should be pointed-out. Firstly, the small sample size might explain some of our lack of association. Secondly, CRF was assessed indirectly. Indeed, there are many concerns regarding the use of running tests as an indicator of CRF in young children. Performance in the growing years can be compromised in many children due to their relative immaturity from a biomechanical and energy efficiency perspective [38], as well as their motivation, especially in girls [39]. However, the easy administration of shuttle-run test and its common use in large scale studies makes it a valuable tool for studying CRF in youth. Furthermore, this study could benefit from additional collected data, such as combined behavioural variables and social background characteristics, which could enhance the outcomes.

\section{CONCLUSION}

In this study it was observed significant relationships between clustered CVD risk factors, fatness indicators, especially percentage of fat, and fitness. After adjustment for fatness, the relationship between fitness and clustered risk factors disappears for all the three adiposity indicators. In conclusion, both fitness and fatness are associated with clustered risk factors by different pathways.

\section{ACKNOWLEDGEMENT}

This study was supported by Foundation for Science and Technology awards SFRH / BD / 15867 / 2005 and PTDC/ DES-72424-2006.

\section{REFERENCES}

[1] Twisk J. Physical activity, physical fitness and cardiovascular health. In: N. Armstrong and W. Van Mechelen, Editors, Paediatric Exercise Science and Medicine. Oxford Oxford University Press, 2000; pp. 253-63.

[2] Genest J, Cohn J. Clustering of cardiovascular risk factors: targeting high-risk individuals. Am J Cardiol 1995; 76: 8A-20A.

[3] Andersen LB, Wedderkopp N, Hansen H, Cooper A, Froberg K. Biological cardiovascular risk factors cluster in Danish children and adolescents: The European Youth Heart Study (EYHS). Prev Med 2003; 37(4): 363-7.

[4] Strong JP, Malcom GT, Newman WP, Oalmann MC. Early lesions of atherosclerosis in childhood and youth: natural history and risk factors. J Am Coll Nutr 1992; 11(Suppl): 51S-54S.

[5] Eisenmann J, Welk G, Wickel E, Blair S. Combined influence of cardiorespiratory fitness and body mass index on cardiovascular disease risk factors among 8-18 year old youth: The Aerobics Center Longitudinal Study. Int J Ped Obes 2007; 2: 66-72.

[6] Ekelund U, Andersen SA, Froberg K, Sardinha LB, Andersen LB, Brage S. Independent associations of physical activity and cardiorespiratory fitness with metabolic risk factors in children: the European Youth Heart Study. Diabetologia 2007; 50: 183240.

[7] Mesa JL, Ortega FB, Ruiz JR, et al. The importance of cardiorespiratory fitness for healthy metabolic traits in children and adolescents: The AVENA Study. J Public Health 2006; 14(3): 178-80.

[8] Eisenmann JC, Wickel EE, Welk GJ, Blair SN. Relationship between adolescent fitness and fatness and cardiovascular disease risk factors in adulthood: the Aerobics Center Longitudinal Study (ACLS). Am Heart 2005; J 149 (1): 46-53.

[9] Brage S, Wedderkopp N, Franks P, Wareham N, Andersen LB, Froberg K. Features of the metabolic syndrome are associated with objectively measured physical activity and fitness in Danish children. Diabetes Care 2004; 27: 2141-8.

[10] Ruiz JR, Ortega FB, Meusel D, Harro M, Oja P, Sjostrom M. Cardiorespiratory fitness is associated with features of metabolic risk factors in children. Should cardiorespiratory fitness be assessed in a European health monitoring system? The European Youth Heart Study. J Public Health 2006; 94-102.

[11] Ruiz J, Castro-Pinero J, Artero E, et al. Predictive validity of health-related fitness in youth: a systematic review. Br J Sports Med 2009; Doi: 0.1136/bjsm.2008.056499.

[12] Castillo-Garzon M, Ruiz J, Ortega F, Gutierrez-Sainz A. A Mediterranean diet is not enough for health: physical fitness is an important additional contributor to health for the adults of tomorrow. World Rev Nutr Diet 2007; 97: 114-38.

[13] Andersen LB, Sardinha LB, Froberg K, Riddoch CJ, Page AS, Anderssen SA. Fitness, fatness and clustering of cardiovascular risk factors in children from Denmark, Estonia and Portugal: the European Youth Heart Study. Int J Pediatr Obes 2008; 3(Suppl 1): 58-66.

[14] Ribeiro JC, Guerra S, Oliveira J, et al. Physical activity and biological risk clustering in pediatric population. Prev Med 2004; 39(3): 546-601.

[15] Shemesh T, Rowley KG, Shephard M, Piers LS, O'Dea K. Agreement between laboratory results and on-site pathology testing using Bayer DCA2000+ and Cholestech LDX point-of-care methods in remote Australian Aboriginal communities. Clin Chim Acta 2006; 367(1-2): 69-76.

[16] U.S. Department of Health and Human Services, PHS. NHANES III Anthropometric Procedures Video. U.S. Government Printing Office Stock Number 017-022-01335-5. Washington, D.C.: U.S. GPO, Public Health Service 1996.

[17] Heyward V. Advanced Fitness Assessment and exercise prescription; 2nd ed. Human Kinetics Champaign, IL 1991.

[18] Slaughter M, Lohman T, Boileau R, et al. Skinfold equations for estimation of body fatness in children and youth. Hum Biol 1988; 60: 709-23. 
[19] Tanner, J. Growth at Adolescence: with a General Consideration of Effects of Hereditary and Environmental Factors Upon Growth and Maturation From Birth to Maturity, $2^{\text {nd }}$ ed. United Kingdom: Blackwell Scientific Publishers 1962.

[20] Mota J, Guerra S, Leandro C, Ribeiro J, Duarte J. Association of maturation, sex, and body fat in cardio respiratory fitness. Am J Hum Biol 2002; 14: 707-12.

[21] Léger L, Lambert J. A maximal multistage $20 \mathrm{~m}$ shuttle run test to predict $\mathrm{VO}_{2} \max$. Eur J Apll Physiol 1982; 49:1-5.

[22] FITNESSGRAM - The Cooper Institute for Aerobic Research. The prudential fitness gram: technical reference manual. In: Morrow JFK, Ed. US. The Cooper Institute for Aerobics Research 1994.

[23] Cooper Institute for Aerobics Research. FITNESSGRAM test administration manual. Champaign, IL: Human Kinetics 1999.

[24] Ahmaidi S, Collomp K, Caillaud C, Préfaut C. The effect of shuttle run test protocol and resulting lactacidemia on maximal velocity and maximal oxygen uptake during the shuttle run exercise test. Eur. J Appl Physiol 1992; 65: 475-9.

[25] Armstrong N, Welsman J. Young people and physical activity. Oxford: Oxford Medical Publications 1997.

[26] Psarra G, Nassis GP, Sidossis LS. Short-term predictors of abdominal obesity in children. Eur J Public Health 2006; 16(5): 520-5.

[27] Eckel RH, Grundy SM, Zimmet PZ. The metabolic syndrome. Lancet 2005; 356: 1415-28.

[28] de Ferrati SD, Gauvreau K, Ludwing DS, Neufeld EJ, Newburger JW, Rifai N. Prevalence of the metabolic syndrome in American adolescents: findings from the Third National Health and Nutrition Examination Survey. Circulation 2004; 110: 2494-7.

[29] Andersen LB, Harro M, Sardinha LB, et al. Physical activity and clustered cardiovascular risk factors: a cross-sectional study (The European Youth Heart Study). Lancet 2006; 368 (9532): 299-304.

[30] Anderssen SA, Cooper A, Riddoch C, et al. Low cardiorespiratory fitness is a strong predictor for clustering of cardiovascular disease risk factors in children independent of country, age and sex. Eur $\mathbf{J}$ Cardiovasc Prev Rehabil 2007; 14: 526-31.

[31] Aires L, Santos R, Silva P, et al. Daily differences in patterns of physical activity among overweight/obese children engaged in a physical activity program. Am J Hum Biol 2007; 19 (6):871-7.

[32] Moran A, Jacobs D, Steinberger J, et al. Insulin resistance during puberty: results from clamp studies in 357 children. Diabetes 1999; 48: $2039-44$

[33] Imperatore G, Cheng Y, Williams D, Fulton J, Gregg E. Physical activity, cardiovascular fitness, and insulin sensitivity among U.S. adolescents. The National Health and Nutrition Examination Survey, 1999-2002. Diabetes Care 2006; 29(7): 1567-71.

[34] Morrison J, Glueck C, Horn P, Schreiber G, Wang P. Pre-teen insulin resistance predicts weight gain, impaired fasting glucose, and type 2 diabetes at age 18-19y: a 10-y prospective study of black and white girls. Am J Clin Nutr 2008; 88: 778-88.

[35] Boreham C, Twisk J, Murray L, Savage M, Strain J, Cran G. Fitness, fatness, and coronary heart disease risk in adolescents: the Northern Ireland Young Hearts Project. Med Sci Sports Excer 2001; 33(2): 270-4.

[36] Yeaman S, Armstrong J, Bonavaud S, Poinasamy D, Pickersgill L, Halse R. Regulation of glycogen synthesis in human muscles cells. Biochem Soc Trans 2001; 29(4): 537-41.

[37] Dela F, Larsen JJ, Mikines KJ, Ploug T, Petersen LN, Galbo H. Insulin-Stimulated Muscle Glucose Clearance in Patients with Niddm - Effects of One-Legged Physical-Training. Diabetes 1995; 44: $1010-20$.

[38] Ebbeling K, Boileau R, Lohman T, Misner J. Determinants of distance running performance in children and adults. Ped Exerc Sci 1992; 4(1): 36-49.

[39] van Mechelen W, Hlobil $\mathrm{H}$. Validation of two running tests as estimates of maximal aerobic power in children. Eur J Appl Physiol Occup Physiol 1986; 55(5): 503-6.

(C) Martins et al.; Licensee Bentham Open.

This is an open access article licensed under the terms of the Creative Commons Attribution Non-Commercial License (http://creativecommons.org/licenses/by-nc/3.0/) which permits unrestricted, non-commercial use, distribution and reproduction in any medium, provided the work is properly cited. 